\title{
Congestion Control in Wireless Software Defined Networks with Propagation Delay and External Interference: A Robust Control Approach
}

\author{
Xi Hu and Wei Guo \\ National Key Laboratory of Science and Technology on Communications, \\ University of Electronic Science and Technology of China, Chengdu, China \\ Correspondence should be addressed to Xi Hu; huxi027@163.com \\ Received 3 July 2016; Revised 11 October 2016; Accepted 20 October 2016 \\ Academic Editor: Fidel Liberal \\ Copyright $\odot 2016$ X. Hu and W. Guo. This is an open access article distributed under the Creative Commons Attribution License, \\ which permits unrestricted use, distribution, and reproduction in any medium, provided the original work is properly cited.

\begin{abstract}
Wireless Software Defined Network (WSDN) presents a new network architecture where the control plane of forwarding devices is shifted to a centralized controller. It is critical to maximize network throughput and keep the network stable during congestion control. However, stability control is insufficient to achieve these aims in the presence of delay and interference. In this paper, we adopt robust control to tackle these problems. Firstly, an efficient weighted scheduling scheme is proposed to maximize the network throughput. Secondly, a robust control model is presented, which is analyzed by Lyapunov-Krasovskii functionals. The sufficient conditions are formulated by Linear Matrix Inequalities (LMIs). Finally, a numerical simulation is conducted to indicate the effectiveness of the proposed scheme.
\end{abstract}

\section{Introduction}

In Wireless Software Defined Networks (WSDNs), the control plane of the forwarding devices is shifted to a centralized controller [1-4]. The forwarding devices keep connected with the centralized controller for constant monitoring and the controller may proactively feed control policies to the forwarding devices to keep the network stable, which is more beneficial to control and manage wireless network [5]. Due to network congestion caused by excessive data based on network services with the same or lower priorities (known as precedence order) [6, 7], it is essential to effectively control congestion and keep network stable [5, 8], for instance, in the differentiated services (DiffServ) for Quality of Services (QoS) that is a combination of several qualities or properties of a network service. In this case, WSDN cannot achieve the maximal global throughput. Furthermore, it is difficult to maintain stability of a wireless network for a long time. Propagation delay and external interference are two key factors that affect a long time stability control for the stability WSDN. The presence of propagation delay causes increment of network cost and unreliability [9], and changing external interference in wireless environments leads to network instability [10].
The two factors make WSDN more difficult to be achieved by long-time stability controls which are even inadequate for tackling the restabilizaion problem, and we thus adopt robust control to solve it. Note that one has to achieve the maximal global throughput via stability control approaches before reaching the robustness.

Some existing solutions would prefer to analyze a robust control for network congestion in SDNs [11-13]. Reference [11] focuses on robust network architecture without additional delays by establishing a prototype with the topology. The authors in [12] modeled network congestion control model with additional time-delay elements to provide a prominent QoS based on Transmission Control Protocol/Active Queue Management (TCP/AQM) networks. In [13], Entity Title Architecture (ETArch) transport model was proposed to tackle QoS control problem. The prototypes or models are dependently established to analyze network congestion control in the current counterpart methods. Unfortunately, these methods cannot robustly control the global wireless network congestion for a long time in consideration of propagation delay and external interference. In addition, they lack sufficient mathematical support. 
In general, the current researches have three crucial limitations. Firstly, the control policies are not located in the centralized controller but implemented by flow tables in the forwarding devices. Secondly, propagation delay is seldom considered in device-controller pairs during congestion control. Finally, the traditional theories for robust control do not work well in WSDNs.

In this paper, to obtain the robust control with the maximal global throughput in WSDNs, a new robust control model is proposed by using Lyapunov-Krasovskii functionals $[14,15]$. The centralized controller generates control polices and feeds control instructions to the forwarding devices. Thus, the forwarding devices could follow these instructions to adjust the padding waiting time. By defining the controlled state as the difference value between the current state and the optimized state, the robust control problem with propagation delay and external interference can be formulated into a robust $H \infty$ control problem [15]. This robust $H \infty$ control problem is then tackled by Lyapunov-Krasovskii functionals. A theorem is also proposed to give the sufficient conditions for the robust $H \infty$ control in LMIs. Based on the theorem, control policies are proposed. Numerical examples are given to indicate the effectiveness of theoretical analysis.

Our main contributions in this paper are listed as follows:

(i) Presenting a realistic global control strategy and the analysis scheme of delay and interference in WSDNs: scheduling scheme currently implemented in the centralized controller can achieve stability control through the global view of real WSDN with the external interference; additionally, the propagation time-delay is essential in real WSDNs and we define an upper bound of delay as the network propagation delay and analyze its influences in global view of WSDN.

(ii) Proposing a general and effective approach to the global robust congestion problem, by calculating optimized values for network parameters with a weighted fair scheduling scheme, and maintaining that optimized status via transformation of a closedloop network model to a normal robust $H \infty$ control model.

(iii) An interdisciplinary effort is made to construct a robust control strategy by combining the stability analysis theories and congestion control principles of wireless network. Taking advantages of the applicability of Lyapunov-Krasovskii functionals in stability analysis of WSDN, the paper achieves the desired global robust control for network congestion.

The rest of the paper is organized as follows. Section 2 briefly introduces the related work. Section 3 proposes the scheduling model and establishes a robust $H \infty$ control model with propagation delay and external interference in device-controller pairs. Section 4 introduces the problem formulation and some preliminaries. Section 5 models the robust control by using Lyapunov-Krasovskii functionals and obtains sufficient conditions. In Section 6, simulations are given to verify the theoretical analysis. The conclusions are drawn in Section 7.

\section{Related Work}

In the recent years, congestion control in WSDNs has attracted considerable attention. Numerous researches have offered stability and robust control algorithms for congestion control in many wireless network scenarios, for example, WSN and VANET, to achieve robust control for network congestion.

Stability control of network congestion has drawn widespread attention and research interests [16, 17]. Existing work has been proposed to tackle the stability control problem. In [18] an analytic study on the interval service response was presented to evaluate the performance based on black burst mechanism. A scheduling strategy was proposed to establish the WSN dynamical model, which adopted traditional stability control theory to address system state stabilization [19]. In [20], the authors operated multiple sensors scheduling simultaneously, and let these sensors switch randomly according to some optimal probability distribution to obtain the best expected stable state performance.

Robust control has attracted particular interest in the literature for the traditional network control system. Based on the wireless characteristic, robust congestion for network congestion has been applied in many wireless network circumstances, for example, WSN [21], VANET [22, 23], Queueing Network [24], and WSDN [11, 25] In WSDNs, the centralized controller had a global view and is responsible for the control and management of all flow tables at each OpenFlow device [25]. A modified SDN-OpenFlow architecture with general operation logic, Multi-Protocol Label Switch (MPLS) management logic, and QoS management logic, was proposed in [11]. In [26] the authors demonstrated the SDN control model in Wireless Sensor and Actor Networks for resource allocation in tasks processing.

Lyapunov-Krasovskii functionals and Linear Matrix Inequalities (LMIs) method are usually proposed to control network congestion. In [9], the authors provided propagation delay model for traditional load frequency control based on Linear Matrix Inequalities (LMIs). Ho is adopted in $[15,27-$ 30] in Network Control Systems (NCSs) to achieve the robust congestion control.

\section{Model and Analysis}

Figure 1 shows a typical scenario of a WSDN with propagation delay and external interference. The centralized controller is able to collect information from all forwarding devices to deal with network congestion. There are two types of delays occurring in the wireless networks: implementation delay and propagation delay [31]. Comparing to the propagation delay, extremely short implementation delay is ignored and propagation delay is considered to analyze the network model, and they usually are not of the same order. The propagation delay is affected by the control plane transmission. The variability of interference in wireless environments leads to network instability [10]. The centralized controller makes global scheduling for the forwarding devices, and accordingly the influence of internal interference is avoided via the prearrangement of network parameters. The paper thus merely considers the 


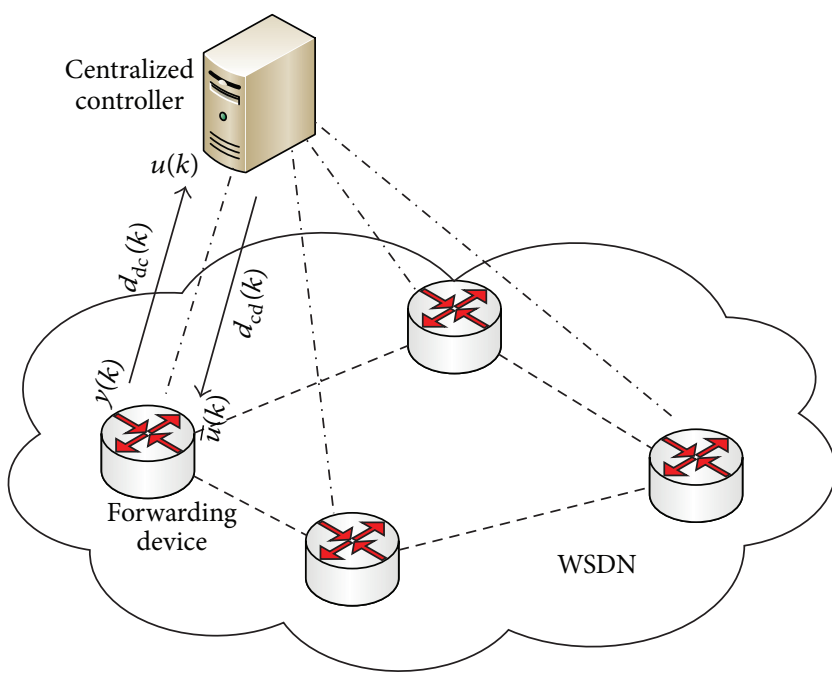

FIGURE 1: A typical scenario of WSDN with the propagation delay $d_{\mathrm{dc}}(k)$ from the forwarding device to the centralized controller (DC) and the one $d_{\mathrm{cd}}(k)$ from centralized controller to the forwarding device (CD).

external interference. There exists an optimized stable state in each forwarding device on stability control for network congestion. Based on the scheduling scheme, the control policies in the centralized controller provide network services to process the data flows on the forwarding devices.

Our goal is to maximize the global network throughput by stability control and keep the global network parameters stable at this optimal state by robust control with propagation delay and external interference. The propagation delay is denoted by $d(k)$, where $k$ is the discrete count number.

The padding waiting time is a key network parameter for robust control in WSDNs. As shown in Figure 2, the processing time is the duration consumed to process a network service. A waiting duration may also be introduced by a scheduling scheme to slow down the forwarding device, and further processing of network services is postponed for this waiting duration. The padding waiting time is defined as the sum of the processing time and the waiting duration. When all forwarding devices are assumed to be at the same processing speeds, minimizing the sum of the padding waiting time may shorten the total service time of all network services and maximize network throughput.

The closed-loop WSDN may be classified into two parts, which are analyzed in the following subsections.

\subsection{A Nonpreemptive Scheduling Scheme Design on Stability} Control for Maximizing the Global WSDN Throughput. A novel nonpreemptive scheduling scheme is proposed, which can be prearranged to stabilize the network parameters of each forwarding device. In order to tackle network congestion problem, in a scheduling problem, one has to find the minimization of the sum of the padding waiting time under the given constraints. Appropriate padding waiting times are arranged for each forwarding device. There exists an optimized scheduling scheme in each forwarding device. The schemes together implement the stability control for network congestion and maximize the global network throughput.

There are $N$ end-to-end network transmission services $E_{i}, i=1,2, \ldots, N$. Each network transmission service is composed of $n_{i}$ point-to-point network transmissions $T_{i j}$, where $j=1, \ldots, n_{i}$ and $n_{i} \leq i$. The point-to-point network transmission $T_{i j}$ obeys a sequential precedence order (QoS/QoE) $T_{i 1} \rightarrow T_{i 2} \rightarrow \cdots \rightarrow T_{i n_{i}}$ that compose the corresponding service $E_{i}$. For convenience, a discretization of event-based sampling is made, so the centralized controller can be considered as an event-based digital controller. There are $K$ forwarding devices $F_{1}, F_{2}, \ldots, F_{K}$ in the closed-loop WSDN that can process these point-to-point network transmissions.

Each point-to-point network transmission $T_{i j}$ is processed within a given duration $p_{i}(k)>0$ with nonpreemption by a forwarding device, where $k$ is the discrete count number. The specific forwarding device is defined as as $u_{i j}(k) \epsilon$ $\left\{F_{k}, k=1,2, \ldots, K\right\}$. Suppose that each forwarding device can process only one $T_{i j}$ at a time, and there are enough buffer spaces in the forwarding devices to store pending network transmissions. Once the forwarding device $k$ starts to process the network transmission services in the fixed duration $p_{i}(k)$, it will not stop until the network transmission services are finished. Each point-to-point network transmission $T_{i j}$ is arranged to a forwarding device $F_{i j}$ in the controller from the previous configuration, such as the wireless routing algorithm. The arrangement had been optimized before the transmission in the ideal network situation. Assume that there are only $k$ levels of the padding waiting time, which is proportional to weight $w_{i}$. Define a scheduling $S=\left(S_{i}(k)\right)$ as the vectors of the padding waiting time for all the network transmission $T_{i j}$ in the forwarding devices $u_{i j}(k)$. Notably, we have $S_{i n_{i}}(k)=0$, where the destination devices finish the endto-end transmission without any more padding waiting time.

Above all, the following arrangement variables is introduced to propose a mathematical description

$$
q_{i}(k)= \begin{cases}1 & \text { if } T_{i j} \text { is arranged in } u_{i j}(k) \\ 0 & \text { otherwise }\end{cases}
$$

For each arrangement $q(k)=\left(q_{i}(k)\right)$, we aim at minimizing the sum of the padding waiting time. When all forwarding devices have the same processing speeds, minimizing the sum of the padding waiting time may shorten total service time of all network services and maximize the global network throughput. Thus, the optimized problem for stability control can be formulated as follows:

$$
\begin{array}{ll}
\min _{k \in \mathbb{N}} & \sum_{i} S_{i}(k) \\
\text { s.t. } & S_{i}(k)+\sum_{m \in u_{i m}(k)} q_{m}(k)\left(p_{m}(k)+S_{m}(k)\right) \\
& \geq S_{i}(k+1) \quad\left(i \geq m=1,2, \ldots, n_{i}-1\right), \\
& \frac{S_{1}}{w_{1}}=\cdots=\frac{S_{i}}{w_{i}}=\cdots=\frac{S_{N}}{w_{N}},
\end{array}
$$




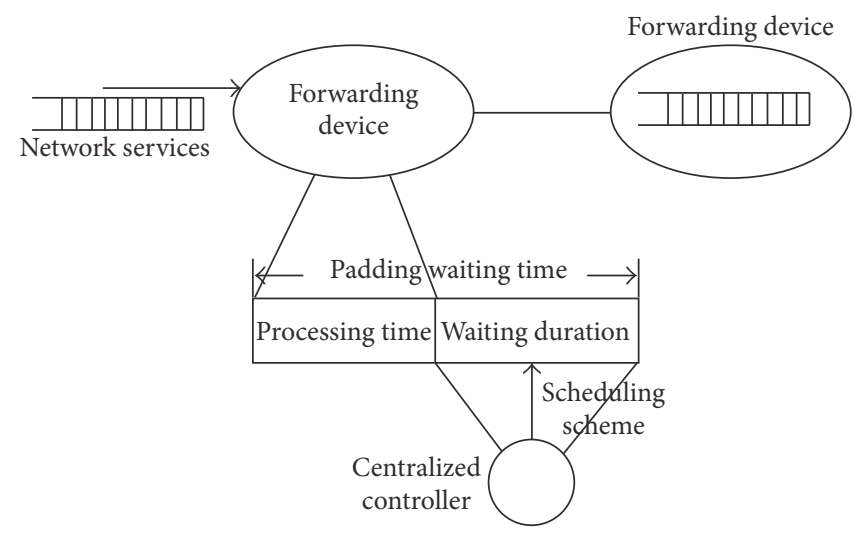

FIGURE 2: The padding waiting time for robust control in WSDNs.

$$
\begin{aligned}
& \sum_{k \in u_{i j}(k)} q_{i}(k) \leq \sum_{k \in T_{i j}} q_{i}(k)=1, \\
& \sum_{i} S_{i n_{i}}(k)=0 \\
& q_{i}(k) \in\{0,1\} \\
& S_{i}(k)>0 .
\end{aligned}
$$

The padding waiting times can be arranged under the constraints before transmission. At the beginning, the optimized problem of minimizing the sum of the padding waiting time can be easily solved and the arrangement proportion is uncomplicated calculated as $S(k)$. At this time, when the optimized state $S(k)$ is calculated and prearranged, the padding waiting time of each forwarding device is stable on stability control at the criterion state $S(k)$.

However, propagation delay and external interference in wireless environments may cause the network unstable. Therefore, the robust control problem is considered to maintain network stability in the presence of propagation delay and external interference.

\subsection{Robust Control Model with Propagation Delay and Exter-} nal Interference. Briefly speaking, when a network transmission service enters the WSDN or it is generated in a forwarding device, it is queued in the buffer and waits for processed and transmitted. If the communication medium becomes free, the scheduling scheme could be nonpreemptively implemented for establishing an end-to-end path in the centralized controller after receiving the advertisements from the forwarding devices. The controller designs the control policy by means of the scheduling scheme and then sends control instructions to adjust the padding waiting time in each forwarding device.

With propagation delay and external interference, the current state $S_{i}(k)$ in each forwarding device pursues the prearranged ideal state $\bar{S}_{i}(k)$ (referring to Section 3.1 ) by robust control, that is, $S_{1}(k) \rightarrow \bar{S}_{1}(k), \ldots, S_{i}(k) \rightarrow \bar{S}_{i}(k), \ldots$, $S_{N}(k) \rightarrow \bar{S}_{N}(k)$. Let $x_{i}(k)=S_{i}(k)-\bar{S}_{i}(k)$ be the error state and $\lim _{k \rightarrow \infty}\left\|\bar{S}_{i}(k)-S_{i}(k)\right\|=0$ for $i=1, \ldots, N$.
According to analyzing the propagation delay in the closed-loop WSDN, the propagation delay from forwarding device to the centralized controller (DC) and the reversed one (CD) are defined as $d_{\mathrm{dc}}(k)$ and $d_{\mathrm{cd}}(k)$, respectively. Suppose that the DC delay $d_{\mathrm{dc}}(k)$ is observable, and the forwarding device receives the feedback message from the controller with the CD delay $d_{\mathrm{cd}}(k)$ in Figure 1. Denote $d(k)=d_{\mathrm{dc}}(k)+d_{\mathrm{cd}}(k)$.

The forwarding devices continually adjust their padding waiting times following the control instructions. Thus, the network services in the forwarding devices constantly achieve nonpreemptive scheduling by means of the advertisements (a packet-in message).

First, the forwarding devices advertise the error state of the network service $x(k)=\left(x_{i}(k)\right)$ to the controller. With the packet-in message, the controller calculates $x(k)$, and the controller classifies the global information of the error state and generates a control policy $u(k)$ to keeping the padding waiting time stable in Figure 1. The control policy needs to restabilize the padding waiting time in the presence of propagation delay and external interference. The controller makes appropriate adjustments of the weighted matrix $B_{u} \in \mathbb{R}^{n \times n}$. Finally, the controller sends a packet-out message, which indicates that the flow originated the packetin message and has been implemented associated with the control instruction. The closed-loop WSDN is accomplished and modeled with propagation delay as

$$
x(k+1)=A x(k)+B_{u} u(k)
$$

where $A$ is the parameters represented the network features that are nonnegative constant matrices with appropriate dimensions.

Thus, the control command $\bar{u}(k)=K x\left(k_{l}\right), K \in \mathbb{R}^{n \times n}$ denotes robust control strength, and the control policies are considered as

$$
\begin{aligned}
x(k+1) & =A x(k)+B_{u} \bar{u}(k), \\
\bar{u}(k) & =K x\left(k_{l}\right),
\end{aligned}
$$


where $d(k)=d_{\mathrm{dc}}(k)+d_{\mathrm{cd}}(k)$, and $\bar{u}(k)$ is the control instructions and $k_{l}$ is the discrete count number. Consider the propagation delay and rewrite $\bar{u}(k)$ as

$$
\begin{aligned}
\bar{u}\left(k_{l}\right) & =\bar{u}\left(k-d\left(k-k_{l}\right)\right)=\bar{u}(k-d(k)) \\
= & K x(k-d(k)), \\
k & \in\left[k_{l}+d(k), k_{l}+d(k+1)\right] .
\end{aligned}
$$

The external interference in this paper is defined as the interference of the duration. Therefore, the external interference is considered as a kind of additive interference, which may lengthen the padding waiting times in the forwarding devices. Then, the closed-loop network model can be formulated into a robust $H \infty$ control model. Simultaneously, the closed-loop WSDN (4) adds the external interference part.

$$
x(k+1)=A x(k)+B_{u} K x(k-d(k))+B_{w} w(k),
$$

where $B_{w}$ is the weight of external interference that is nonnegative constant matrices with appropriate dimensions. For convenience, we assume the external interference is limited energy and duration.

Thus, the robust $H \infty$ control model of the error state with propagation delay and external interference is formulated in the closed-loop WSDN.

\section{Problem Formulations of Robust $H \infty$ Control}

According to the term (4), the robust $H \infty$ control model of the error state with propagation delay and external interference in the closed-loop WSDN is described by

$$
\begin{aligned}
x(k+1) & =A x(k)+B_{u} u(k)+B_{w} w(k), \\
u(k) & =K x(k-d(k)), \\
z(k) & =I x(k),
\end{aligned}
$$

where $x(k)=\left[x_{1}(k), \ldots, x_{n}(k)\right]^{T} \in \mathbb{R}^{n}$ is the error state which denotes the varying value of the padding waiting time between the current state and the ideal state, $u(k)=$ $\left[u_{1}(k), \ldots, u_{n}(k)\right]^{T} \in \mathbb{R}^{n}$ is the control instruction, $w(k)=$ $\left[w_{1}(k), \ldots, w_{n}(k)\right]^{T} \in \mathbb{R}^{n}$ is the external interference of limited energy and duration with covariance matrix $w$ and has expectation zero, $z(k)=\left[z_{1}(k), \ldots, z_{n}(k)\right]^{T}$ as a measurement is the output of the robust control, and $k$ is the discrete count number. Define

$$
\begin{aligned}
A & =\left(a_{i j}\right)_{n \times n}= \begin{cases}a_{i j}=0, & \text { if } i<j \\
0 \leq a_{i j} \leq 1, & \text { if } i \geq j,\end{cases} \\
B_{u} & =\left(b_{i j}\right)_{n \times n}, \\
B_{w} & =\operatorname{diag}\left\{\widehat{b}_{1}, \widehat{b}_{2}, \ldots, \widehat{b}_{n}\right\},
\end{aligned}
$$

and the constant matrix $I \in \mathbb{R}^{n \times n}$. As the aforementioned analysis, $u(k)=K x(k-d(k)), K \in \mathbb{R}^{n \times n}$ denotes robust control strength. $d(k)$ denotes the propagation delay.
Definition 1 (see [29]). For given two positives $d_{m}, d_{M}>$ 0 , the closed-loop WSDN (7) is stable with the external interference $w(k) . d_{m}, d_{M}$ represented its upper and lower bounds, respectively, which satisfies $0<d_{m}<d(k) \leq d_{M}$.

Definition 2. The closed-loop WSDN (7) is said to stable, if there exists a state feedback control instruction $u(k)=$ $K x(k-d(k)), K \in \mathbb{R}^{n \times n}$. Thus, $u(k)$ is said to the robust control policies.

Lemma 3 (Schur complement [32]). Given constant matrices $P, Q, R$, where $P^{T}=P$ and $Q^{T}=Q$, and then the $L M I$ $\left[\begin{array}{cc}{ }_{P} & R \\ R^{T} & -Q\end{array}\right]<0$ is equivalent to the following condition: $Q>0$, $P+R Q^{-1} R^{T}<0$.

\section{Criteria of Robust $H \propto$ Control}

In the following, let $\bar{A}=A-I, \bar{B}=B_{u} K$. Rewrite closed-loop WSDN of the error state (7) into a more compact form as

$$
\begin{aligned}
& y(k)=\bar{A} x(k)+\bar{B} x(k-d(k))+B_{w} w(k), \\
& z(k)=I x(k),
\end{aligned}
$$

where $y(k)=x(k+1)-x(k)$ is the difference state, and

$$
\begin{gathered}
y(k)=\left[x_{1}(k+1)-x_{1}(k), x_{2}(k+1)\right. \\
\left.-x_{2}(k), \ldots, x_{n}(k+1)-x_{n}(k)\right]^{T} .
\end{gathered}
$$

Theorem 4. Consider the robust Ho control model of the error state with propagation delay and external interference in the closed-loop WSDN (9). Given the external interference attenuation level $\gamma$ and positive integers $d_{m}, d_{M}\left(0<d_{m}<\right.$ $\left.d_{M}\right)$. The closed-loop WSDN achieves robust Ho control, if there exist appropriate dimension symmetric positive definite matrices $P>0, Q>0, R>0$, and $S>0, X=\left[\begin{array}{cc}X_{11} & X_{12} \\ * & X_{22}\end{array}\right] \geq$ 0 , and appropriate dimension matrices $N_{1}, N_{2}$, so that the following conditions in (11) hold:

$$
\begin{aligned}
& \Xi=\left[\begin{array}{ccc}
X_{11} & X_{12} & N_{1} \\
* & X_{22} & N_{2} \\
* & * & R
\end{array}\right] \geq 0, \\
& \Omega=\left[\begin{array}{cccccc}
\omega_{11} & \omega_{12} & P B_{w} & \bar{A}^{T} & d_{M} \bar{A}^{T} & I \\
* & \omega_{22} & 0 & \bar{B}^{T} & d_{M} \bar{B}^{T} & 0 \\
* & * & -\gamma^{2} I & B_{w}^{T} & d_{M} B_{w}^{T} & 0 \\
* & * & * & -P^{-1} & 0 & 0 \\
* & * & * & * & -d_{M} R^{-1} & 0 \\
* & * & * & * & * & -I
\end{array}\right]<0,
\end{aligned}
$$


where

$$
\begin{aligned}
\omega_{11}= & P \bar{A}+\bar{A}^{T} P+Q+\left(d_{M}-d_{m}+1\right) S+N_{1}+N_{1}^{T} \\
& +d_{M} X_{11}, \\
\omega_{12}= & P \bar{B}-N_{1}+N_{2}^{T}+d_{M} X_{12}, \\
\omega_{22}= & -Q-S-N_{2}-N_{2}^{T}+d_{M} X_{22} .
\end{aligned}
$$

Proof. We firstly define

$$
y(l)=x(l+1)-x(l)
$$

Then, we obtain

$$
\begin{aligned}
x(k+1) & =x(k)+y(k), \\
x(k)-x(k-d(k))-\sum_{l=k-d(k)}^{k-1} y(l) & =0 .
\end{aligned}
$$

In the closed-loop network, the Lyapunov-Krasovskii functionals can be expressed by

$$
\begin{aligned}
V(k) & =\sum_{n=1}^{4} V_{n}(k), \\
V_{1}(k) & =x^{T}(k) P x(k), \\
V_{2}(k) & =\sum_{j=k-d(k)}^{k-1} x^{T}(j) Q x(j), \\
V_{3}(k) & =\sum_{\theta=-d_{M}+1}^{0} \sum_{j=k-1+\theta}^{k-1} y^{T}(j) R y(j), \\
V_{4}(k) & =\sum_{j=-d_{M}+1} \sum_{l=k+j-1}^{k-1} x^{T}(l) S x(l),
\end{aligned}
$$

where $P=P^{T}>0, Q=Q^{T}>0, R=R^{T}>0$, and $S=S^{T}>$ 0 are positive definite symmetric matrices. Define $\triangle V(k)=$ $V(k+1)-V(k)$; thus

$$
\begin{aligned}
\triangle V_{1}(k)= & x^{T}(k+1) P x(k+1)-x^{T}(k) P x(k) \\
= & 2 x^{T}(k) P y(k)+y^{T}(k) P y(k), \\
\triangle V_{2}(k)= & \sum_{j=k-d(k)+1}^{k} x^{T}(j) Q x(j) \\
& -\sum_{j=k-d(k)}^{k-1} x^{T}(j) Q x(j) \\
= & x^{T}(k) Q x(k) \\
& -x^{T}(k-d(k)) Q x(k-d(k)),
\end{aligned}
$$

$$
\begin{aligned}
\triangle V_{3}(k)= & d_{M} y^{T}(k) R y(k)-\sum_{l=k-d_{M}}^{k-1} y(l) R y(l) \\
\leq & d_{M} y^{T}(k) R y(k)-\sum_{l=k-d(k)}^{k-1} y^{T}(l) R y(l), \\
\triangle V_{4}(k)= & \left(d_{M}-d_{m}+1\right) x^{T}(k) S x(k) \\
& -\sum_{l=k-d_{M}}^{k-d_{m}} x^{T}(l) S x(l) \\
\leq & \left(d_{M}-d_{m}+1\right) x^{T}(k) S x(k) \\
& -x^{T}(k-d(k)) S x(k-d(k)) .
\end{aligned}
$$

For any appropriate dimension matrix $N_{i}(i=1,2)$, we have

$$
\begin{aligned}
0= & 2\left[x^{T}(k) N_{1}+x^{T}(k-d(k)) N_{2}\right] \\
& \times\left[x(k)-x(k-d(k))-\sum_{l=k-d(t)}^{k-1} y(l)\right] .
\end{aligned}
$$
we get

For an appropriate dimension matrix $X=\left[\begin{array}{cc}X_{11} & X_{12} \\ * & X_{22}\end{array}\right] \geq 0$,

$$
\begin{aligned}
0 & \leq \sum_{l=k-d_{M}}^{k-1} \zeta_{1}^{T}(k) X \zeta_{1}(k)-\sum_{l=k-d(k)}^{k-1} \zeta_{1}^{T}(k) X \zeta_{1}(k) \\
& =d_{M} \zeta_{1}^{T}(k) X \zeta_{1}(k)-\sum_{l=k-d(t)}^{k-1} \zeta_{1}^{T}(k) X \zeta_{1}(k) .
\end{aligned}
$$

Thus, from (16) to (18), we have

$$
\begin{aligned}
\Delta V(k) \leq & \zeta_{2}^{T}(k)\left\{\Lambda+\Pi_{1}^{T}\left(P+d_{M} R\right) \Pi_{1}\right\} \zeta_{2}(k) \\
& -\sum_{l=k-d(k)}^{k-1} \zeta_{3}^{T}(k, l) \Xi \zeta_{3}(k, l) \\
& +\gamma^{2} w^{T}(k) w(k),
\end{aligned}
$$

with

$$
\begin{aligned}
\zeta_{1}(k) & =\left[\begin{array}{ll}
x^{T}(k) & x^{T}(k-d(k))
\end{array}\right]^{T}, \\
\zeta_{2}(k) & =\left[\begin{array}{lll}
x^{T}(k) & x^{T}(k-d(k)) & w^{T}(k)
\end{array}\right]^{T}, \\
\zeta_{3}(k, l) & =\left[\begin{array}{lll}
x^{T}(k) & x^{T}(k-d(k)) y^{T}(l)
\end{array}\right]^{T}, \\
\Lambda & =\left[\begin{array}{ccc}
\varphi_{11} & \varphi_{12} & P B_{w} \\
* & \varphi_{22} & 0 \\
* & * & -\gamma^{2} I
\end{array}\right], \\
\Pi_{1} & =\left[\begin{array}{lll}
\bar{A} & \bar{B} & B_{w}
\end{array}\right]^{T}, \\
\Pi_{2} & =\left[\begin{array}{lll}
I & 0 & 0
\end{array}\right] .
\end{aligned}
$$


Defining $\Theta=\Lambda+\Pi^{T} P \Pi+d_{M} \Pi^{T} R \Pi$ and using Schur complement lemma (Lemma 3), the LMIs in (19) can make inequalities $\Theta<0$ true. Then there exists a positive scalar $\varepsilon>0$ such that $\Theta<\varepsilon I<0$. Therefore, it follows that $\Delta V(k) \leq$ $-\varepsilon\|x(k)\|^{2}<0 \forall x(k) \neq 0$.

Considering $w(t) \neq 0$ and Schur complement lemma, following the inequalities (11), we have

$$
\begin{aligned}
\triangle & V(k)+z^{T}(k) z(k)-\gamma^{2} w^{T}(k) w(k) \\
& \leq \xi^{T}(k) \Omega \xi(k)<0 .
\end{aligned}
$$

Sum $k$ from 0 to $\infty$ with the initialization of $V(0)=0$; we can obtain

$$
\sum_{k=0}^{\infty}\left\{z^{T}(k) z(k)-\gamma^{2} w^{T}(k) w(k)\right\}<0 .
$$

Based on the Lyapunov-Krasovskii functionals, the robust $H \infty$ control model of the error state with propagation delay and external interference in the closed-loop WSDN can achieve robust $H \infty$ control $J<0$ with desired $H \infty$ performance index $\left\|T_{w z}(z)\right\|_{\infty}<\gamma$ following (11).

The proof is complete.

The parameters of desired robust $H \infty$ control can be obtained through LMI in MATLAB, and the performance evaluations are given through SIMULINK in MATLAB.

\section{Simulation Results}

In this section, a numerical simulation is conducted to indicate the effectiveness of the proposed scheme in WSDNs and the control policies are designed given in Theorem 4.

In actual WSDNs, QoS mechanism is the typical instance as the precedence order of network service contained integrated service and differentiated service. Usually, there are eight priority levels in the QoS mechanism, defined from 0 to 7 with 0 being the highest. Denote $x_{i}(k), i=0,1, \ldots, 7$, as these eight priority levels in WSDNs. Consider the robust $H \infty$ control model (9) with different network parameters to clearly demonstrate different QoS control policies in the centralized controller as follows: $B_{w}=\operatorname{diag}\{0.2,0.1,0.31,0.51$, $0.11,0.21,0.31,0.41\}$ and

A

$$
=\left[\begin{array}{cccccccc}
0.22 & 0 & 0 & 0 & 0 & 0 & 0 & 0 \\
0.36 & 0.15 & 0 & 0 & 0 & 0 & 0 & 0 \\
0.65 & 0 & 0.3 & 0 & 0 & 0 & 0 & 0 \\
0.1 & 0 & 0.51 & 0.22 & 0 & 0 & 0 & 0 \\
0.63 & 0.14 & 0 & 0.25 & 0.33 & 0 & 0 & 0 \\
0.36 & 0 & 0.25 & 0 & 0 & 0.47 & 0 & 0 \\
0.36 & 0.94 & 0.74 & 0.51 & 0 & 0.65 & 0.45 & 0 \\
0.31 & 0.26 & 0 & 0.59 & 0 & 0.3 & 0.1 & 0.14
\end{array}\right],
$$

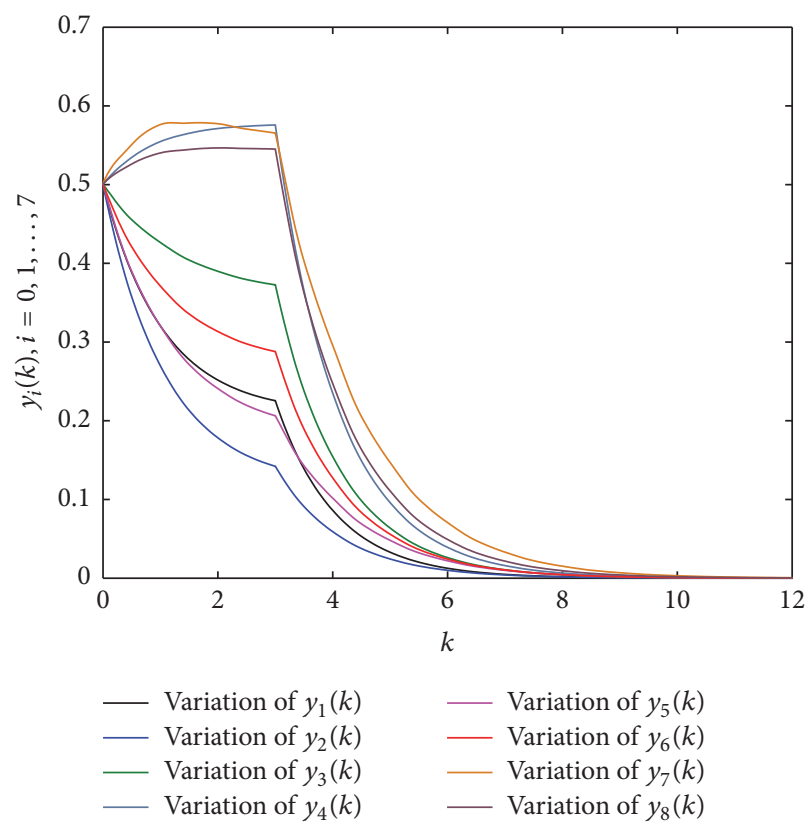

FIGURE 3: The variations of $y_{i}(k)$ (at eight priority levels) with the initial state $y_{i}(0)=0.5, i=0,1, \ldots, 7$, in the presence of propagation delay and external interference.

where $A$ denotes the relationship between $x(k+1)$ and $x(k)$. Define

$$
A=\left(a_{i j}\right)= \begin{cases}a_{i j}=0, & \text { if } i<j \\ 0 \leq a_{i j} \leq 1, & \text { if } i \geq j\end{cases}
$$

that means the precedence orders. In actual wireless network environment, before starting its execution, the flow with lower priority needs to wait for finishing the completions of all flow queues with nonlower priorities in the forwarding device $k$. The padding waiting time consists of the probability weight of every nonlower priority.

6.1. Effectiveness Verification of the Proposed Scheme in WSDNs. According to Theorem 4, there exists a feasible solution to LMIs (11). We use the different state $y_{i}(k)=x_{i}(k+$ $1)-x_{i}(k)$ to reflect the approaching results of error state. That is, $y_{i}(t)$ means the error state of the padding waiting time between adjacent time intervals.

Suppose the control strength $K=-0.8 I$, the control policy $B_{u}=A$, and the initial conditions are 0.5 and 1 , respectively. Two scenarios with the different initial states are considered to make a comparison. Suppose that a step function represents the external interference with limited energy and duration to make the simulation tractable.

Notably, the error states may increase or decrease based on the different initial state. However, the difference states $y(k)$ can maintain system stable in the presence of propagation delay and external inferences, as shown in Figures 3 and 4 . The different initial states still make convergence in the presence of the step external interference, which represents that all error states $x(k)$ reach an agreement 


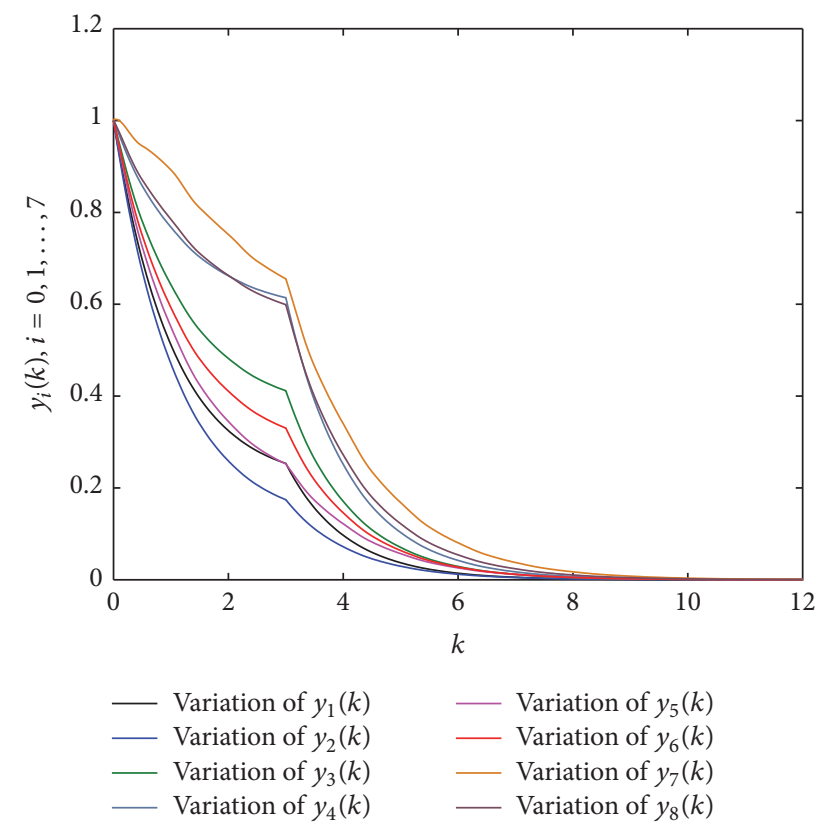

FIGURE 4: The variations of $y_{i}(k)$ (at eight priority levels) with the initial state $y_{i}(0)=1, i=0,1, \ldots, 7$, in the presence of propagation delay and external interference.

by the centralized controlling. Thus, this simulation can be conducted to indicate the effectiveness of the proposed scheme in WSDNs.

The final convergence of error states in Figures 3 and 4 shows that the robust control has stabilized the state divergence aroused by the propagation delay and external interference, which occurred beyond the stable initial status conducted by traditional stability control. In other words, the robust control has successfully enhanced the effect of the stabilization by traditional stability control.

6.2. Design of Control Policy on Robust Ho Control in WSDNs. This section introduces the design of the control policy based on the proposed scheme in WSDNs. We select the intermediate priority $i=4$ in the simulation. Figures 5 and 6 show the variations of the error state under the different control strength $K$. The initial state is $y_{4}(0)=0.5$ (Figure 5), while $y_{4}(0)=1$ (Figure 6). Compared with the different initial states and control strength $K$, it is notable that a tighter control results in the smaller width.

Therefore, the appropriate adjustments of QoS control policy can easily be designed in the controller. The control policy can be designed to control the width measurement under different initial error states.

\section{Conclusion}

This paper have adopted robust control to tackle the problems of maximizing network throughput and keeping the network stable during congestion control in WSDNs. Firstly, an efficient nonpreemptive scheduling scheme has been proposed to maximize the global network throughput. Secondly,

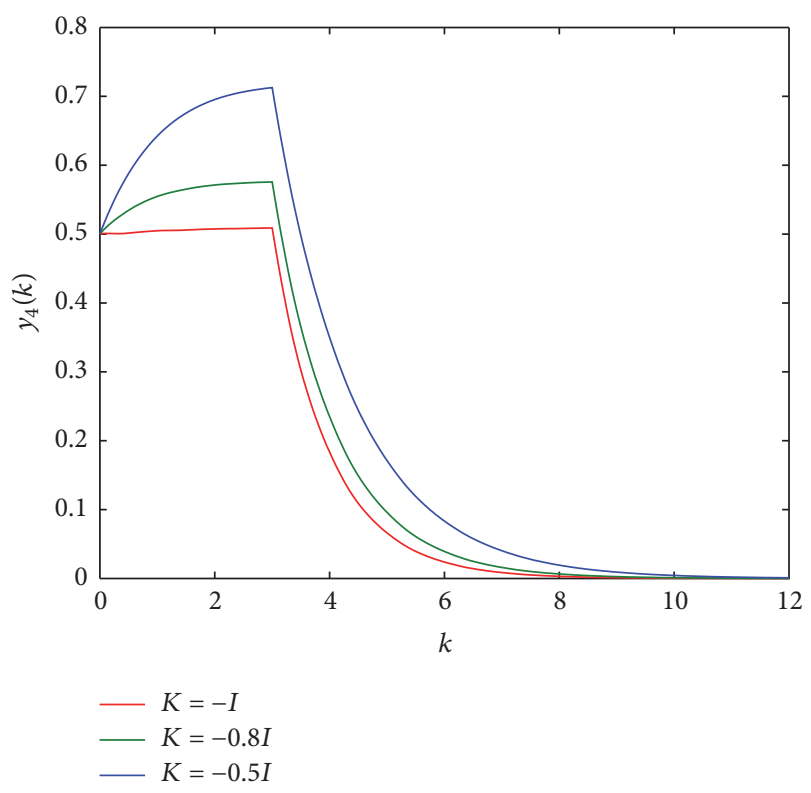

FIGURE 5: The variations of $y_{4}(k)$ with the initial state $y_{4}(0)=0.5$ and different control strength $K$ in the presence of propagation delay and external interference.

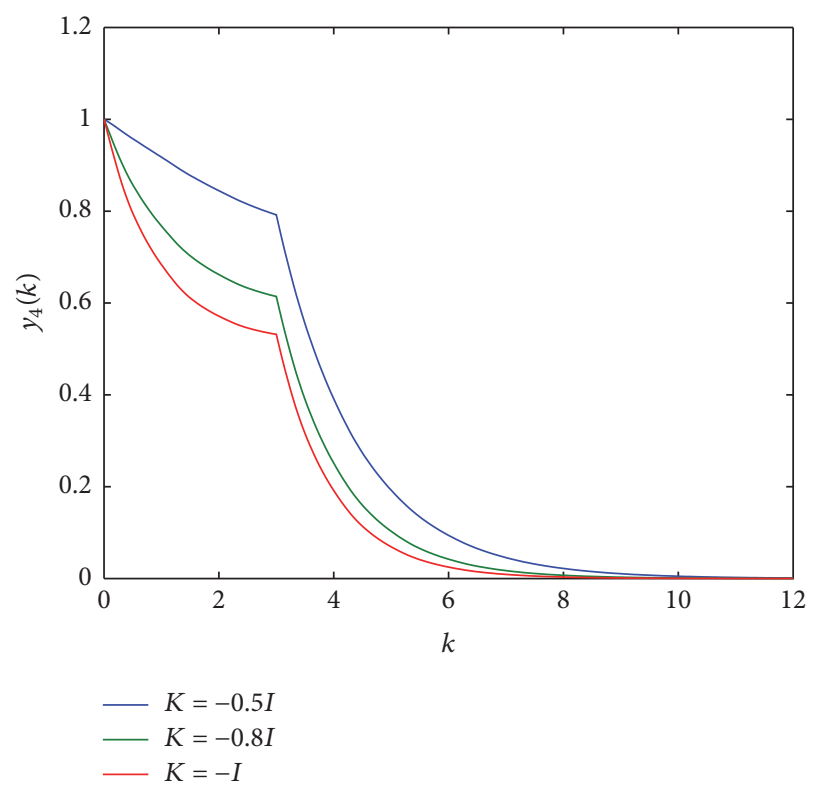

FIGURE 6: The variations of $y_{4}(k)$ with the initial state $y_{4}(0)=1$ and different control strength $K$ in the presence of propagation delay and external interference.

a robust control model with propagation delay and external interference is presented by using Lyapunov-Krasovskii functionals. The sufficient conditions have been formulated by LMIs. Finally, the numerical simulation has been conducted to indicate the effectiveness of the proposed scheme.

Future studies should explore the impact of implementation delays and stochastic external interference on the performance of robust control for network congestion in WSDNs. Delays are ubiquitous in wireless networks while 
frequently causing stability problems. The approach that we have presented would be extended to develop more complex algorithms and be applied in other wireless fields.

\section{Competing Interests}

The authors declare that they have no competing interests.

\section{Acknowledgments}

This work is supported by the 863 project (Grant no. 2014AA01A701); the National Natural Science Foundation of China (Grant nos. 61271168, 61471104).

\section{References}

[1] S. Agarwal, M. Kodialam, and T. V. Lakshman, "Traffic engineering in software defined networks," in Proceedings of the 32nd IEEE Conference on Computer Communications (IEEE INFOCOM '13), pp. 2211-2219, Turin, Italy, April 2013.

[2] L. F. Muller, R. R. Oliveira, M. C. Luizelli, L. P. Gaspary, and M. P. Barcellos, "Survivor: an enhanced controller placement strategy for improving SDN survivability," in Proceedings of the IEEE Global Communications Conference (GLOBECOM '14), pp. 1909-1915, IEEE, Austin, Tex, USA, December 2014.

[3] Z. Y. Su, T. Wang, Y. Xia, and M. Hamdi, "FlowCover: lowcost flow monitoring scheme in software defined networks," in Proceedings of the IEEE Global Communications Conference (GLOBECOM '14), pp. 1956-1961, Austin, Tex, USA, December 2014.

[4] N. McKeown, T. Anderson, H. Balakrishnan et al., "OpenFlow: enabling innovation in campus networks," ACM SIGCOMM Computer Communication Review, vol. 38, no. 2, pp. 69-74, 2008.

[5] S. Tomovic, N. Prasad, and I. Radusinovic, "SDN control framework for QoS provisioning," in Proceedings of the 22nd Telecommunications Forum Telfor (TELFOR '14), pp. 111-114, Belgrade, Serbia, November 2014.

[6] V. Esmaeelzadeh, E. S. Hosseini, R. Berangi, and O. B. Akan, "Modeling of rate-based congestion control schemes in cognitive radio sensor networks," Ad Hoc Networks, vol. 36, pp. 177$188,2016$.

[7] T. Feng, J. Bi, and K. Wang, "Allocation and scheduling of network resource for multiple control applications in SDN," China Communications, vol. 12, no. 6, Article ID 7122483, pp. 85-95, 2015.

[8] M. Caria, A. Jukan, and M. Hoffmann, "A performance study of network migration to SDN-enabled Traffic Engineering," in Proceedings of the IEEE Global Communications Conference (GLOBECOM '13), pp. 1391-1396, Atlanta, Ga, USA, December 2013.

[9] X. F. Yu and K. Tomsovic, "Application of linear matrix inequalities for load frequency control with communication delays," IEEE Transactions on Power Systems, vol. 19, no. 3, pp. 1508-1515, 2004.

[10] L. Yang, X.-P. Guan, C.-N. Long, and X.-Y. Luo, "Feedback stabilization over wireless network using adaptive coded modulation," International Journal of Automation and Computing, vol. 5, no. 4, pp. 381-388, 2008.

[11] F. López-Rodríguez and D. R. Campelo, "A robust SDN network architecture for service providers," in Proceedings of the
IEEE Global Communications Conference (GLOBECOM '14), pp. 1903-1908, December 2014.

[12] H. Chibana, M. Yoshino, M. Tadokoro, D. Murayama, K. Suzuki, and R. Kubo, "Disturbance-observer-based active queue management with time delay using software-defined networking controller," in Proceedings of the 41st Annual Conference of the IEEE Industrial Electronics Society (IECON '15), pp. 001049-001054, Yokohama, Japan, November 2015.

[13] J. Castillo, A. Neto, F. Silva et al., "Additions to the ETArch control plane to support multimedia QoS-guaranteed content transport over OpenFlow-enabled SDN future internet systems," in Proceedings of the IEEE Globecom Workshops (GC Wkshps '14), pp. 172-177, IEEE, 2014.

[14] Y. S. Moon, P. Park, W. H. Kwon, and Y. S. Lee, "Delaydependent robust stabilization of uncertain state-delayed systems," International Journal of Control, vol. 74, no. 14, pp. 14471455, 2001.

[15] D. Yue, Q.-L. Han, and J. Lam, "Network-based robust $H_{\infty}$ control of systems with uncertainty," Automatica, vol. 41, no. 6, pp. 999-1007, 2005.

[16] R. Johari and D. K. H. Tan, "End-to-end congestion control for the internet: delays and stability," IEEE/ACM Transactions on Networking, vol. 9, no. 6, pp. 818-832, 2001.

[17] G. Bansal and J. B. Kenney, "Achieving weighted-fairnessin message rate-based congestion control for DSRC systems," in Proceedings of the IEEE 5th International Symposium on Wireless Vehicular Communications (WiVeC '13), pp. 1-5, Dresden, Germany, June 2013.

[18] N. Boughanmi, Y.-Q. Song, and E. Rondeau, "Priority and adaptive QoS mechanism for wireless networked control systems using IEEE 802.15.4," in Proceedings of the 36th Annual Conference of the IEEE Industrial Electronics Society (IECON '10), pp. 2134-2141, IEEE, Glendale, Calif, USA, November 2010.

[19] C. X. Qu, H. S. Li, and W. Chen, "Virtual queue based distributed data traffic scheduling in WSNs for distributed voltage control in smart micro-grid," in Proceedings of the 10th IEEE International Conference on Intelligent Sensors, Sensor Networks and Information Processing (ISSNIP '15), pp. 1-6, Singapore, April 2015.

[20] V. Gupta, T. H. Chung, B. Hassibi, and R. M. Murray, "On a stochastic sensor selection algorithm with applications in sensor scheduling and sensor coverage," Automatica, vol. 42, no. 2, pp. 251-260, 2006.

[21] C. Sergiou, V. Vassiliou, and A. Paphitis, "Hierarchical Tree Alternative Path (HTAP) algorithm for congestion control in wireless sensor networks," Ad Hoc Networks, vol. 11, no. 1, pp. 257-272, 2013.

[22] S. Djahel and Y. Ghamri-Doudane, "A robust congestion control scheme for fast and reliable dissemination of safety messages in VANETs," in Proceedings of the IEEE Wireless Communications and Networking Conference (WCNC '12), pp. 2264-2269, Paris, France, April 2012.

[23] X. Shen, X. Cheng, R. Zhang, B. Jiao, and Y. Yang, "Distributed congestion control approaches for the ieee 802.11p vehicular networks," IEEE Intelligent Transportation Systems Magazine, vol. 5, no. 4, pp. 50-61, 2013.

[24] R. Pedarsani, J. Walrand, and Y. Zhong, "Robust scheduling and congestion control for flexible queueing networks," in Proceedings of the International Conference on Computing, Networking and Communications (ICNC '14), pp. 467-471, February 2014.

[25] L.-H. Huang, H.-J. Hung, C.-C. Lin, and D.-N. Yang, "Scalable and bandwidth-efficient multicast for software-defined 
networks," in Proceedings of the IEEE Global Communications Conference (GLOBECOM '14), pp. 1890-1896, IEEE, Austin, Tex, USA, December 2014.

[26] W. Li, D. Liu, B. Zhu, X. Wei, W. Xiao, and L. Yang, "SDN control model for intelligent task execution in wireless sensor and actor networks," in Proceedings of the IEEE 83rd Vehicular Technology Conference (VTC Spring '16), pp. 1-5, Nanjing, China, May 2016.

[27] F. Rasool and S. K. Ngaung, "Robust $H_{\infty}$ dynamic output feedback control of networked control systems with congestion control," International Journal of Systems Science, vol. 46, no. 10, pp. 1854-1864, 2015.

[28] F. Rasool, S. K. Nguang, and C.-M. Lin, "Robust $H_{\infty}$ state feedback control of networked control systems with congestion control," Circuits, Systems, and Signal Processing, vol. 32, no. 6, pp. 2761-2781, 2013.

[29] T. Wang, T. Li, X. Yang, and S. Fei, "Cluster synchronization for delayed Lur'e dynamical networks based on pinning control," Neurocomputing, vol. 83, pp. 72-82, 2012.

[30] Q. Song and Z. Zhao, "Cluster, local and complete synchronization in coupled neural networks with mixed delays and nonlinear coupling," Neural Computing and Applications, vol. 24, no. 5, pp. 1101-1113, 2014.

[31] X. Lin, L. Hong, and R. Jingyu, "Research on predictive control of stochastic network-induced delay for network control systems," in Proceedings of the in Chinese Control and Decision Conference (CCDC '11), pp. 929-932, Mianyang, China, May 2011.

[32] S. Boyd, L. El Ghaoui, E. Feron, and V. Balakrishnan, Linear Matrix Inequalities in System and Control Theory, vol. 15 of SIAM Studies in Applied Mathematics, Society for Industrial and Applied Mathematics, Philadelphia, Pa, USA, 1994. 

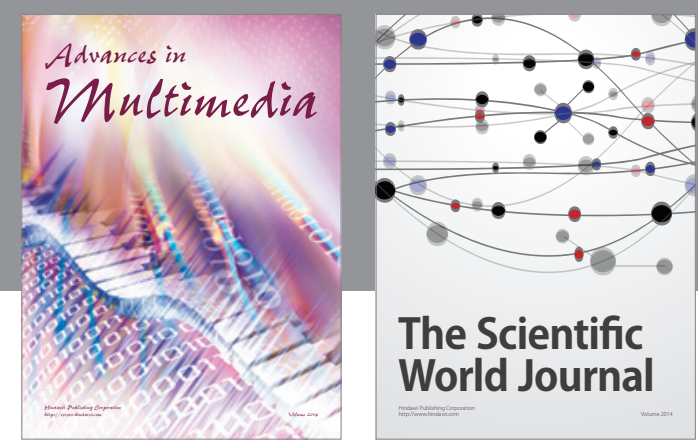

The Scientific World Journal
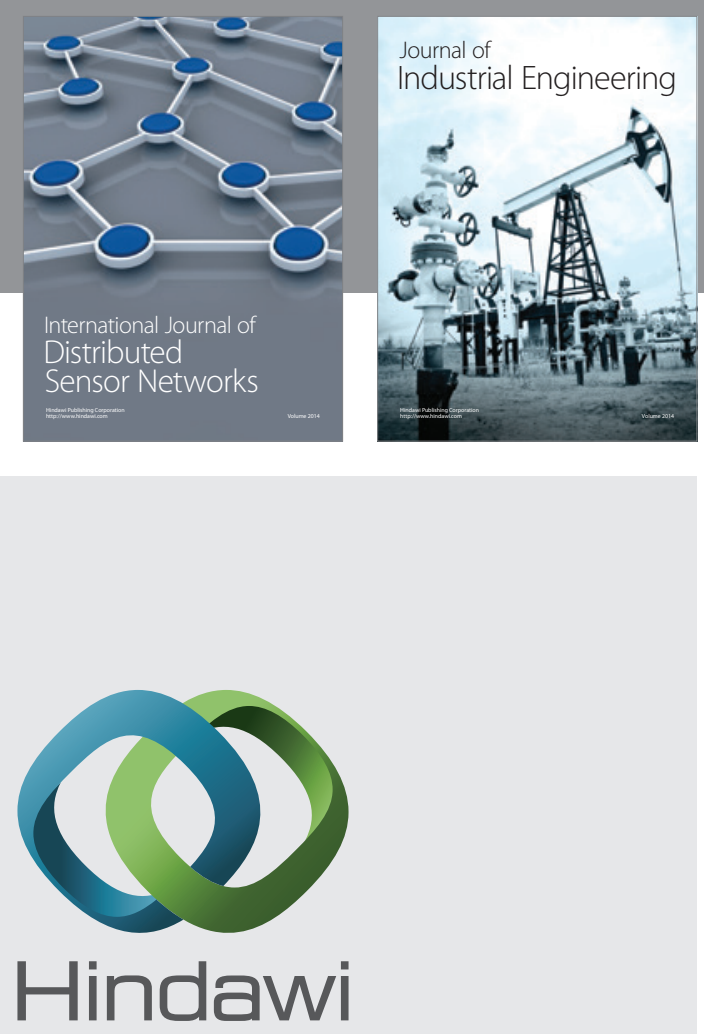

Submit your manuscripts at

http://www.hindawi.com

\section{Computer Networks} and Communications
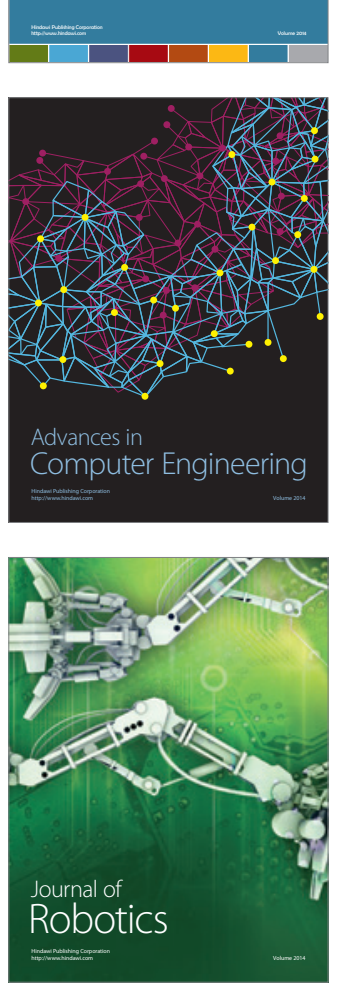
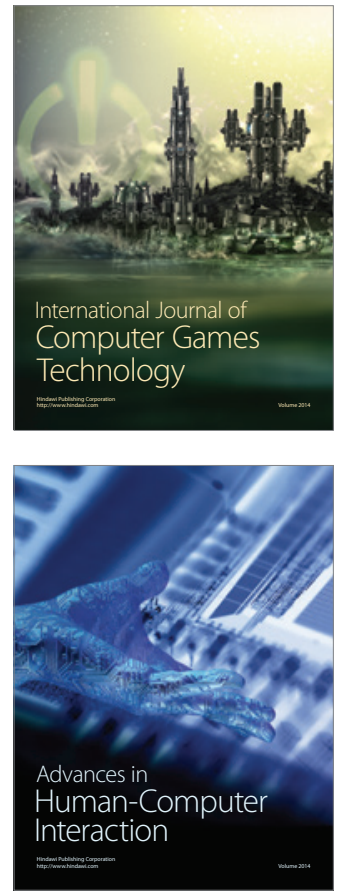
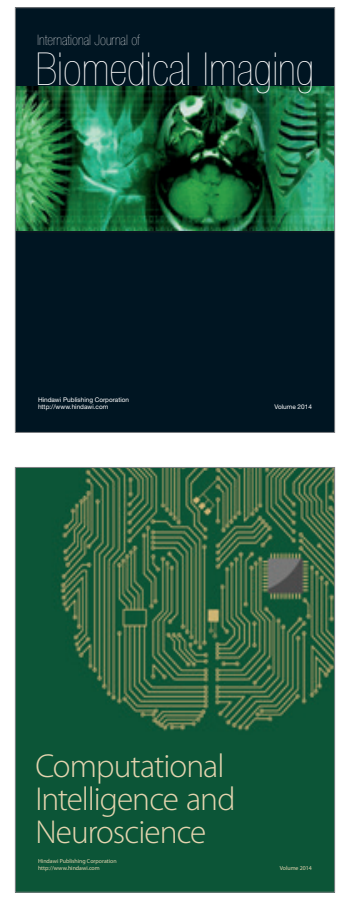
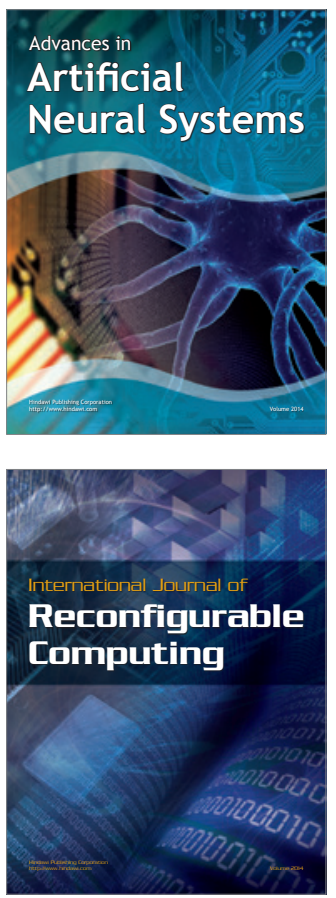
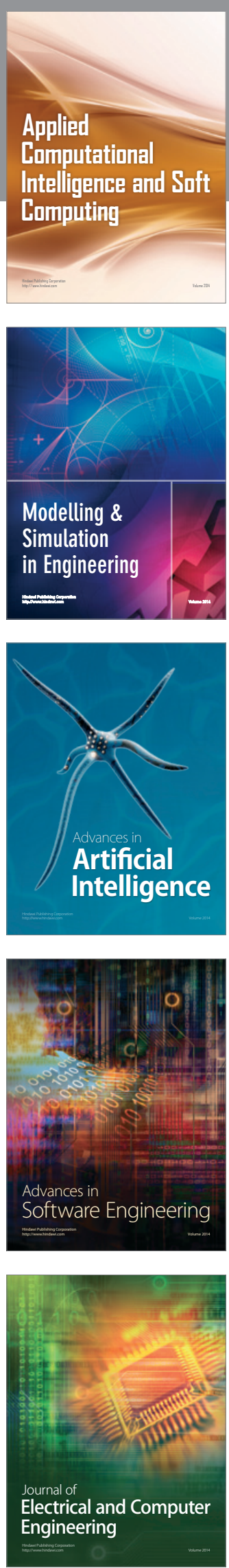